Research Article

\title{
Pinning Synchronization of Nonlinearly Coupled Complex Dynamical Networks on Time Scales
}

\author{
Fang-Di Kong $\mathbb{D}^{1,2}$ \\ ${ }^{1}$ College of Electrical and Information Engineering, Lanzhou University of Technology, Lanzhou 730050, China \\ ${ }^{2}$ Department of Applied Mathematics, Lanzhou University of Technology, Lanzhou 730050, China \\ Correspondence should be addressed to Fang-Di Kong; kongfd@lut.edu.cn
}

Received 19 December 2019; Accepted 21 May 2020; Published 8 July 2020

Academic Editor: Soheil Salahshour

Copyright $\odot 2020$ Fang-Di Kong. This is an open access article distributed under the Creative Commons Attribution License, which permits unrestricted use, distribution, and reproduction in any medium, provided the original work is properly cited.

In this paper, we study the synchronization problem for nonlinearly coupled complex dynamical networks on time scales. To achieve synchronization for nonlinearly coupled complex dynamical networks on time scales, a pinning control strategy is designed. Some pinning synchronization criteria are established for nonlinearly coupled complex dynamical networks on time scales, which guarantee the whole network can be pinned to some desired state. The model investigated in this paper generalizes the continuous-time and discrete-time nonlinearly coupled complex dynamical networks to a unique and general framework. Moreover, two numerical examples are given for illustration and verification of the obtained results.

\section{Introduction}

Complex networks are an important part of our daily life and in nature, due to many systems in the real world which can be modeled by complex dynamical networks, such as the Internet, World Wide Web, and food webs [1]. There have been a lot of researches on complex networks and neural networks (see, for example, [2-8] and references therein). Synchronization of complex dynamical network has been a hot topic in the past decades [9-16]. When considering synchronization problem of complex dynamical network, the controlled synchronization problem of complex dynamical network is significant. In recent years, the pinning synchronization of complex dynamical networks, which means the network to achieve desired synchronization by applying control to a small fraction of network nodes, has become a topic of great interest; see [17-34]. In particular, Liu and Chen [16] investigated the global synchronization for nonlinearly coupled complex networks. Further, they investigated pinning synchronization for continuous-time nonlinearly coupled networks; see [32]. In [33], a pinning control scheme was developed for continuous-time nonlinearly coupled complex dynamical network, while the results were extended to discrete-time case. In [34], the synchronization of continuous-time dynamical networks with nonlinearly coupling function was considered.

In real life, the time domains do not always match the known continuous-time intervals or discrete integer time domains. From practical point of view, it is important to study complex dynamic networks on general time domains. This is the starting point of the present investigation. Recently, synchronization of complex dynamical networks on time scales has attracted considerable attention [35-43], which contains not only synchronization of continuoustime and discrete-time complex dynamical networks but also some continuous-time intervals accompanying some discrete moments.

Motivated by the aforementioned discussions, the synchronization of nonlinearly coupled complex dynamical networks on time scales by applying pinning control scheme will be investigated. The objective in this paper is driving the whole network to some desired state by pinning control strategy. By investigating pinning controlled networks on time scales, some sufficient conditions are presented to guarantee the realization of pinning synchronization for nonlinearly coupled complex dynamical networks on time scales. The main contributions of this paper are listed as follows: 
(i) The model investigated in this paper generalizes the continuous-time and discrete-time nonlinearly coupled complex dynamical networks to a unique and general framework. Therefore, the obtained results include continuous-time and discrete-time nonlinearly coupled complex dynamical networks as special cases. Moreover, the model investigated in this paper is more general. Our results can be applied to investigate pinning synchronization of nonlinearly coupled complex dynamical networks on a mixed time domain

(ii) Linearly coupled complex dynamical networks on time scale are included as a special case of the present work

The rest of this paper is organized as follows. Some foundational knowledge about time scales and some notations and supporting lemmas are simply outlined in Section 2. In Section 3, the pinning synchronization problem of nonlinearly coupled complex dynamical networks on time scales is formulated. In Section 4, the main theorems and some corollaries are established. In Section 5, two numerical examples are given to verify the effectiveness of our results. Finally, conclusions are provided in Section 6.

\section{Preliminaries}

In this section, we will present some foundational knowledge about time scales and some notations and lemmas which are needed later.

2.1. Foundational Knowledge on Time Scales. Throughout this paper, $\mathbb{N}, \mathbb{Z}$, and $\mathbb{R}$ denote the sets of positive integers, integers, and real numbers, respectively. A time scale is defined as a nonempty closed subset of $\mathbb{R}$ and denoted by $\mathbb{T}$.

Definition 1 (see $[44,45]$ ). Let $t \in \mathbb{T}$. Define the forward jump operator $\sigma: \mathbb{T} \longrightarrow \mathbb{T}$ by $\sigma(t)=\inf \{\tau \in \mathbb{T}: \tau>t\}$, while the backward jump operator $\rho: \mathbb{T} \longrightarrow \mathbb{T}$ is defined by $\rho(t)=$ $\sup \{\tau \in \mathbb{T}: \tau<t\}$. In this definition, we put inf $\varnothing=\sup \mathbb{T}$ and $\sup \varnothing=\inf \mathbb{T}$, where $\varnothing$ denotes the empty set. If $\sigma(t)>$ $t$, we say that $t$ is right-scattered, while if $\rho(t)<t$, we say that $t$ is left-scattered. Also, if $t<\sup \mathbb{T}$ and $\sigma(t)=t$, then $t$ is called right-dense, and if $t>\inf \mathbb{T}$ and $\rho(t)=t$, then $t$ is called left-dense. The graininess function $\mu: \mathbb{T} \longrightarrow[0,+\infty)$ is defined by $\mu(t):=\sigma(t)-t$. The set $\mathbb{T}^{\kappa}$ is derived from the time scale $\mathbb{T}$ as follows: if $\mathbb{T}$ has a left-scattered maximum $m$, then $\mathbb{T}^{\kappa}=\mathbb{T} \backslash\{m\}$. Otherwise, $\mathbb{T}^{\kappa}=\mathbb{T}$.

Definition 2 (see [44]). Let $f: \mathbb{T} \longrightarrow \mathbb{R}$. Define the function $f^{\sigma}: \mathbb{T} \longrightarrow \mathbb{R}$ by $f^{\sigma}(t)=f(\sigma(t))$ for all $t \in \mathbb{T}$, i.e., $f^{\sigma}=f \circ \sigma$.

Definition 3 (see $[44,45]$ ). Assume that $f: \mathbb{T} \longrightarrow \mathbb{R}$ and $t \in$ $\mathbb{T}^{\mathcal{K}}$, then $f$ is called $\Delta$-differentiable at the point $t$ if there exists $\theta \in \mathbb{R}$ such that for any given $\varepsilon>0$, there is an open neighborhood $U$ of the point $t$ such that

$$
\left|f^{\sigma}(t)-f(s)-\theta(\sigma(t)-s)\right| \leq \varepsilon|\sigma(t)-s|, \quad s \in U
$$

In this case, $\theta$ is called the $\Delta$-derivative of $f$ at the point $t$ and we denote it by $\theta=f^{\Delta}(t)$. Moreover, we say that $f$ is $\Delta$-differentiable (or in short: differentiable) on $\mathbb{T}^{\mathcal{K}}$ provided $f^{\Delta}(t)$ exists for all $t \in \mathbb{T}^{\kappa}$. The function $f^{\Delta}: \mathbb{T}^{\kappa} \longrightarrow \mathbb{R}$ is called the $\Delta$-derivative of $f$ on $\mathbb{T}^{\kappa}$. If $F^{\Delta}(t)=f(t), t \in \mathbb{T}^{\kappa}$, then for any $a, b \in \mathbb{T}$, the integral is defined as follows:

$$
\int_{a}^{b} f(t) \Delta t=F(b)-F(a)
$$

Remark 4. If $\mathbb{T}=\mathbb{R}$, then $f^{\sigma}(t)=f(t), f^{\Delta}(t)=f^{\prime}(t)$ is the usual derivative, and $\int_{a}^{b} f(t) \Delta t=\int_{a}^{b} f(t) d t$ is the usual integral. If $\mathbb{T}=\mathbb{Z}$, then $f^{\sigma}(t)=f(t+1)$ is the forward shift, $f^{\Delta}(t)=\Delta f(t)=f(t+1)-f(t)$ is the usual forward difference and $\int_{a}^{b} f(t) \Delta t=\sum_{t=a}^{b-1} f(t)(a<b)$.

Lemma 5 (see $[44,45])$. If $f, g: \mathbb{T} \longrightarrow \mathbb{R}$ are differentiable at $t \in \mathbb{T}^{\kappa}$, then

$$
(f g)^{\Delta}(t)=f^{\Delta}(t) g(t)+f^{\sigma}(t) g^{\Delta}(t)=f(t) g^{\Delta}(t)+f^{\Delta}(t) g^{\sigma}(t)
$$

Lemma 6 (see $[44,45]$ ). If $f: \mathbb{T} \longrightarrow \mathbb{R}$ is differentiable at $t$ $\in \mathbb{T}^{\kappa}$, then $f^{\sigma}(t)=f(t)+\mu(t) f^{\Delta}(t)$.

Definition 7 (see $[44,45]$ ). A function $f: \mathbb{T} \longrightarrow \mathbb{R}$ is called $r d$ -continuous provided it is continuous at right-dense points in $\mathbb{T}$ and its left-sided limits exist (finite) at left-dense points in $\mathbb{T}$. The set of these functions is denoted by $C_{r d}(\mathbb{T} ; \mathbb{R})$.

Definition 8 (see $[44,45])$. We say that a function $p: \mathbb{T} \longrightarrow \mathbb{R}$ is regressive (positive regressive) provided

$$
1+\mu(t) p(t) \neq 0 \quad(1+\mu(t) p(t)>0), \quad \text { for all } t \in \mathbb{T}^{\kappa}
$$

holds. The set of all regressive (positive regressive) and $r d$ -continuous functions is denoted by $\mathscr{R}(\mathbb{T} ; \mathbb{R})\left(\mathscr{R}^{+}(\mathbb{T} ; \mathbb{R})\right)$.

Definition 9 (see $[44])$. If $p \in \mathscr{R}(\mathbb{T} ; \mathbb{R})$, then we define the exponential function by

$$
e_{p}(t, s)=\exp \left(\int_{s}^{t} \xi_{\mu(\tau)}(p(\tau)) \Delta \tau\right), \quad \text { for all } s, t \in \mathbb{T},
$$

where the cylinder transformation $\xi_{h}(z)$ is defined by

$$
\xi_{h}(z)= \begin{cases}\frac{1}{h} \log (1+z h), & h>0, \\ z, & h=0,\end{cases}
$$

where Log is the principal logarithm function.

Lemma 10 (see [44]). Let $t_{0} \in \mathbb{T}, y, f \in C_{r d}(\mathbb{T} ; \mathbb{R})$ and $p \in$ $\mathscr{R}^{+}(\mathbb{T} ; \mathbb{R})$. Then,

$$
y^{\Delta}(t) \leq p(t) y(t)+f(t), \quad \text { for all } t \in \mathbb{T}
$$


implies

$$
y(t) \leq y\left(t_{0}\right) e_{p}\left(t, t_{0}\right)+\int_{t_{0}}^{t} e_{p}(t, \sigma(\tau)) f(\tau) \Delta \tau, \quad \text { for all } t \in \mathbb{T} .
$$

Lemma 11 (see [43]). For fixed $t_{0} \in \mathbb{T}$, if $p<0$ and $p \in \mathscr{R}^{+}$ $(\mathbb{T} ; \mathbb{R})$, then $e_{p}\left(t, t_{0}\right) \longrightarrow 0$ as $t \longrightarrow+\infty, t \in \mathbb{T}$.

Let $A$ be an $m \times n$-matrix-valued function on $\mathbb{T}$. We say that $A$ is differentiable on $\mathbb{T}$ provided each entry of $A$ is differentiable on $\mathbb{T}$. In this case, we put $A^{\Delta}(t)=\left(a_{i j}^{\Delta}(t)\right)_{1 \leq i \leq m, 1 \leq j \leq n}$, where $A=\left(a_{i j}(t)\right)_{1 \leq i \leq m, 1 \leq j \leq n}$.

Lemma 12 (see [44]). If a matrix-valued function $A$ is differentiable at $t \in \mathbb{T}^{\kappa}$, then $A^{\sigma}(t)=A(t)+\mu(t) A^{\Delta}(t)$.

Lemma 13 (see [44]). Suppose that $A$ and $B$ are differentiable $n \times n$-matrix-valued functions on $\mathbb{T}$. Then,

(1) $(A+B)^{\Delta}=A^{\Delta}+B^{\Delta}$

(2) $(\alpha A)^{\Delta}=\alpha A^{\Delta}$, where $\alpha$ is a constant

(3) $(A B)^{\Delta}=A^{\Delta} B+A^{\sigma} B^{\Delta}=A B^{\Delta}+A^{\Delta} B^{\sigma}$

2.2. Notations and Supporting Lemmas. For each interval $\square$ of

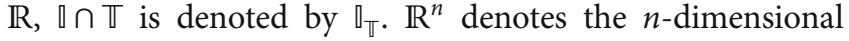
Euclidean space with the Euclidean norm $\|\cdot\| . \mathbb{R}^{m \times n}$ denotes the set of all $m \times n$ real matrices. Let $I_{N} \in \mathbb{R}^{N \times N}$ be the $N$ dimensional identity matrix, $\operatorname{diag}\left(d_{1}, d_{2}, \cdots, d_{N}\right)$ indicate the diagonal matrix with diagonal entries $d_{1}$ to $d_{N}, A^{T}$ be the transpose of matrix $A$, and $\lambda_{\min }(\cdot)$ and $\lambda_{\max }(\cdot)$ represent the minimum eigenvalue and the maximum eigenvalue of a real symmetric matrix. For a symmetric matrix $P \in \mathbb{R}^{n \times n}$, write $P>0 \quad(P<0, \quad P \geq 0$, and $P \leq 0$, respectively $)$ if $P$ is positive definite (negative definite, positive semidefinite, and negative semidefinite, respectively). For square matrices $A$ and $B$, the notation $A \geq B(A \leq B)$ means that $A-B$ is a positive semidefinite (negative semidefinite) matrix. The symbol $\otimes$ denotes the Kronecker product.

Lemma 14 (see [46]). For matrices $A, B, C$, and $D$ with appropriate dimensions, we have the following properties:

(1) $(\alpha A) \otimes B=A \otimes(\alpha B)=\alpha(A \otimes B)$, where $\alpha$ is a constant

(2) $(A+B) \otimes C=A \otimes C+B \otimes C$

(3) $(A \otimes B)^{T}=A^{T} \otimes B^{T}$

(4) If $A$ and $B$ are symmetric, then $A \otimes B$ is symmetric

(5) For square matrices $A$ and $B$, every eigenvalue of $A \otimes B$ arises as a product of eigenvalues of $A$ and $B$

Lemma 15 (see [47]). Let $U=\left(\alpha_{i j}\right)_{N \times N}, M \in \mathbb{R}^{n \times n}, x=$ $\left(x_{1}^{T}, x_{2}^{T}, \cdots, x_{N}^{T}\right)^{T}$, where $x_{i}=\left(x_{i 1}, x_{i 2}, \cdots, x_{i n}\right)^{T} \in \mathbb{R}^{n}$ and $y=$
$\left(y_{1}^{T}, y_{2}^{T}, \cdots, y_{N}^{T}\right)^{T}$, where $y_{i}=\left(y_{i 1}, y_{i 2}, \cdots, y_{i n}\right)^{T} \in \mathbb{R}^{n} \quad(i=1,2$, $\cdots, N)$. If $U=U^{T}$, and each row sum of $U$ is zero, then

$$
x^{T}(U \otimes M) y=-\sum_{1 \leq i<j \leq N} \alpha_{i j}\left(x_{i}-x_{j}\right)^{T} M\left(y_{i}-y_{j}\right) .
$$

Lemma 16 (see [26]. Suppose $A=\left(a_{i j}\right)_{N \times N}$ is a real symmetric and irreducible matrix, in which $a_{i j} \geq 0(j \neq i)$ and $a_{i i}=-\sum_{j=1, j \neq i}^{N} a_{i j}$, nonzero matrix $D=\operatorname{diag}\left(d_{1}, d_{2}, \cdots, d_{N}\right)$ satisfies $d_{i} \geq 0(1 \leq i \leq N)$. Let $B=A-D$. Then, all the eigenvalues of $B$ are less than 0 .

Lemma 17 (see [48]). If $A, B \in \mathbb{R}^{m \times m}$ are symmetric, $x \in \mathbb{R}^{m}$, $x \neq 0$ (m-dimensional zero vector) and $0<a, b \in \mathbb{R}$, then

(1) $A^{k}$ is symmetric for $k \in \mathbb{N}$

(2) $A$ is positive definite $\Leftrightarrow-A$ is negative definite

(3) $A$ is positive definite $\Leftrightarrow$ there exists a positive definite matrix $C \in \mathbb{R}^{m \times m}$ such that $A=C C$, that is, $C$ is a real square root of $A$

(4) $\lambda_{\min }(A) \leq x^{T} A x / x^{T} x \leq \lambda_{\max }(A)$

(5) $\lambda_{\max }(a A+b B) \leq a \lambda_{\max }(A)+b \lambda_{\max }(B)$

(6) if $\lambda$ is the eigenvalue of $A$, then $\lambda^{k}$ is the eigenvalue of $A^{k}, k \in \mathbb{N}$

\section{Problem Formulations}

Throughout the rest of the paper, let $\mathbb{T}$ be a time scale with $0 \in \mathbb{T}$ and $\sup \mathbb{T}=+\infty$. In this section, the nonlinearly coupled complex dynamical network on the time scale $\mathbb{T}$ will be introduced.

In general, the dynamic for each isolated (uncoupled) node of the dynamical network can be described as

$$
s^{\Delta}(t)=f(s(t)), \quad t \in[0,+\infty)_{\mathbb{T}},
$$

where $s(t)=\left(s_{1}(t), s_{2}(t), \cdots, s_{n}(t)\right)^{T} \in \mathbb{R}^{n}, s^{\Delta}$ is the $\Delta$-derivative of $s$ on $[0,+\infty)_{\mathbb{T}}, f: \mathbb{R}^{n} \longrightarrow \mathbb{R}^{n}$ is continuous and of such a nature that existence and uniqueness of solutions to dynamic equation (10) subject to $s(0)=s_{0} \quad\left(s_{0} \in \mathbb{R}^{n}\right)$ as well as their dependence on initial values is guaranteed.

Suppose that the dynamical network consists of $N$ identical nodes, with each node being an $n$-dimensional dynamical system. Then, the nonlinearly coupled dynamical network can be described by

$$
\begin{gathered}
x_{i}^{\Delta}(t)=f\left(x_{i}(t)\right)+c \sum_{j=1}^{N} g_{i j} h\left(x_{j}(t)\right), \quad t \in[0,+\infty)_{\mathbb{T}}, \\
i=1,2, \cdots, N
\end{gathered}
$$

where $x_{i}(t)=\left(x_{i 1}(t), x_{i 2}(t), \cdots, x_{\text {in }}(t)\right)^{T} \in \mathbb{R}^{n}$ is the state vector of the $i$ th node at time $t$; the constant $c>0$ represents the 
coupling strength of network; the nonlinearly coupled function $h: \mathbb{R}^{n} \longrightarrow \mathbb{R}^{n}$ is continuous, which satisfies standard assumptions on existence and uniqueness of solutions to dynamic equation (11) subject to $x(0)=x_{0} \quad\left(\mathrm{x}_{0} \in \mathbb{R}^{n}\right)$ as well as their dependence on initial values; the coupling configuration matrix $G=\left(g_{i j}\right) \in \mathbb{R}^{N \times N}$ represents the topological structure of the complex network and is defined as follows: if there exists a connection between the node $i$ and the node $j(i \neq j)$, then $g_{i j}=g_{j i}=1$; otherwise, $g_{i j}=g_{j i}=0$, and $g_{i i}=-\sum_{j=1, j \neq i}^{N} g_{i j}=-\sum_{j=1, j \neq i}^{N} g_{j i}, i=1,2, \cdots, N$. Suppose that network (11) is connected in the sense of having no isolated clusters, which means that the coupling configuration matrix $G$ is irreducible.

Suppose that $s(t)$ is a solution of the uncoupled system (10). In order to synchronize the network (11) to the objective state $s(t)$, we will design a pinning control scheme, if the network (11) cannot synchronize to the objective state $s$ $(t)$ without control. Without loss of generality, we add the controllers to the first $l$ nodes. Hence, we have the pinningcontrolled network as follows:

$$
\begin{gathered}
x_{i}^{\Delta}(t)=f\left(x_{i}(t)\right)+c \sum_{j=1}^{N} g_{i j} h\left(x_{j}(t)\right)+u_{i}(t), \quad t \in[0,+\infty)_{\mathbb{T}}, \\
i=1,2, \cdots, N
\end{gathered}
$$

with the feedback controllers given by

$u_{i}(t)=-c d_{i}\left(h\left(x_{i}(t)\right)-h(s(t))\right), \quad t \in[0,+\infty)_{\mathbb{T}}, i=1,2, \cdots, N$,

where $d_{i}=d>0, \quad(i=1,2, \cdots, l), \quad d_{i}=0, \quad(i=l+1, l+2, \cdots$ $, N)$. Define the matrix: $D=\operatorname{diag}\left(d_{1}, d_{2}, \cdots, d_{l}, \cdots, d_{N}\right)$.

Definition 18 (see [30]). The network (12) is said to be synchronized by pinning control, if

$$
\lim _{t \rightarrow \infty}\left\|x_{i}(t)-s(t)\right\|=0, \quad i=1,2, \cdots, N
$$

We get the following error dynamical network by letting $z_{i}(t)=x_{i}(t)-s(t) \in \mathbb{R}^{n}(i=1,2, \cdots, N)$ :

$$
\begin{aligned}
z_{i}^{\Delta}(t)= & f\left(x_{i}(t)\right)-f(s(t))+c \sum_{j=1}^{N} g_{i j}\left(h\left(x_{j}(t)\right)-h(s(t))\right) \\
& -c d_{i}\left(h\left(x_{i}(t)\right)-h(s(t))\right), \quad t \in[0,+\infty)_{\mathbb{T}}, i=1,2, \cdots, N .
\end{aligned}
$$

It follows from Lemma 16 that the symmetric matrix $G-D$ is negative definite, and so the maximal eigenvalue $\lambda_{\max }(G-D)<0$. From Lemma $17((1)-(3))$, it is easy to prove that the matrix $(G-D)^{2}$ is symmetric positive definite.

\section{Pinning Synchronization Criteria for Nonlinearly Coupled Complex Dynamical Networks on Time Scales}

In order to derive the synchronization criteria for the pinning-controlled network (12), we make the following assumptions:

(A1) (see [18]). The function $f: \mathbb{R}^{n} \longrightarrow \mathbb{R}^{n}$ is assumed to satisfy Lipschitz condition, that is, there exists a constant $l_{1}>0$ such that

$$
\|f(x)-f(y)\| \leq l_{1}\|x-y\| \text { holds for any } x, y \in \mathbb{R}^{n} .
$$

(A2) (see $[9,21,49])$. There exists a constant $l_{2}>0$, such that

$(x-y)^{T}(h(x)-h(y)) \geq l_{2}(x-y)^{T}(x-y), \quad$ for any $x, y \in \mathbb{R}^{n}$.

(A3). There exists a constant matrix $K \in \mathbb{R}^{n \times n}$, such that

$$
\begin{aligned}
& (f(x)-f(y))^{T}(h(x)-h(y)) \geq(x-y)^{T} K(x-y), \\
& \quad \text { for any } x, y \in \mathbb{R}^{n} .
\end{aligned}
$$

(A4) (see [18]). The function $h: \mathbb{R}^{n} \longrightarrow \mathbb{R}^{n}$ is assumed to satisfy Lipschitz condition, that is, there exists a constant $l_{3}>0$ such that

$$
\|h(x)-h(y)\| \leq l_{3}\|x-y\| \text { holds for any } x, y \in \mathbb{R}^{n} \text {. }
$$

We have the following theorems and corollaries, which give some sufficient conditions to guarantee synchronization of the network (12) by pinning control.

Theorem 19. Suppose that assumptions (A1), (A2), (A3), and (A4) hold. If there exists $\theta: \mathbb{T} \longrightarrow \mathbb{R}$ with $\theta \in \mathscr{R}^{+}(\mathbb{T} ; \mathbb{R})$ and $\lim _{t \rightarrow \infty} e_{\theta}(t, 0)=0$ such that the following condition is satisfied:

$$
\begin{aligned}
& 2 l_{1} I_{n N}+2 c l_{2}\left[(G-D) \otimes I_{n}\right]+\mu(t)\left[l_{1}^{2} I_{n N}\right. \\
& \left.\quad+2 c((G-D) \otimes K)+c^{2} l_{3}^{2} \lambda_{\max }\left((G-D)^{2}\right) I_{n N}\right] \leq \theta(t) I_{n N} \\
& \quad t \in[0,+\infty)_{\mathbb{T}},
\end{aligned}
$$

then, the network (12) is synchronized by pinning control.

Proof. Consider the Lyapunov function $V(t)=\sum_{i=1}^{N} z_{i}^{T}(t)$ $z_{i}(t), t \in[0,+\infty)_{\mathbb{T}}$. By Lemmas $5,6,12$, and 13 , calculating the $\Delta$-derivative of $V(t)$ along the trajectories of the error dynamical network (15), one has 


$$
\begin{aligned}
& V^{\Delta}(t)=\sum_{i=1}^{N}\left[\left(z_{i}^{T}\right)^{\Delta}(t) z_{i}(t)+\left(z_{i}^{T}\right)^{\sigma}(t) z_{i}^{\Delta}(t)\right] \\
& =\sum_{i=1}^{N}\left\{\left(z_{i}^{T}\right)^{\Delta}(t) z_{i}(t)+\left[z_{i}^{T}(t)+\mu(t)\left(z_{i}^{T}\right)^{\Delta}(t)\right] z_{i}^{\Delta}(t)\right\} \\
& =\sum_{i=1}^{N}\left[2 z_{i}^{T}(t) z_{i}^{\Delta}(t)+\mu(t)\left(z_{i}^{\Delta}(t)\right)^{T} z_{i}^{\Delta}(t)\right] \\
& =\sum_{i=1}^{N}\left\{2 z _ { i } ^ { T } ( t ) \left[\left(f\left(x_{i}(t)\right)-f(s(t))\right)+c \sum_{j=1}^{N} g_{i j}\left(h\left(x_{j}(t)\right)\right.\right.\right. \\
& \left.-h(s(t)))-c d_{i}\left(h\left(x_{i}(t)\right)-h(s(t))\right)\right] \\
& +\mu(t)\left[\left(f\left(x_{i}(t)\right)-f(s(t))\right)^{T}\right. \\
& +c\left(\sum_{j=1}^{N} g_{i j}\left(h\left(x_{j}(t)\right)-h(s(t))\right)\right)^{T} \\
& \left.-c d_{i}\left(h\left(x_{i}(t)\right)-h(s(t))\right)^{T}\right]\left[\left(f\left(x_{i}(t)\right)\right.\right. \\
& -f(s(t)))+c \sum_{j=1}^{N} g_{i j}\left(h\left(x_{j}(t)\right)-h(s(t))\right) \\
& \left.\left.-c d_{i}\left(h\left(x_{i}(t)\right)-h(s(t))\right)\right]\right\} \\
& =\sum_{i=1}^{N}\left\{2 z_{i}^{T}(t)\left(f\left(x_{i}(t)\right)-f(s(t))\right)+2 c z_{i}^{T}(t) \sum_{j=1}^{N} g_{i j}\right. \\
& \cdot\left(h\left(x_{j}(t)\right)-h(s(t))\right)-2 c d_{i} z_{i}^{T}(t)\left(h\left(\mathrm{x}_{i}(t)\right)-h(s(t))\right) \\
& +\mu(t)\left[\left(f\left(x_{i}(t)\right)-f(s(t))\right)^{T}\left(f\left(x_{i}(t)\right)-f(s(t))\right)\right. \\
& +c\left(f\left(x_{i}(t)\right)-f(s(t))\right)^{T} \sum_{j=1}^{N} g_{i j}\left(h\left(x_{j}(t)\right)-h(s(t))\right) \\
& -c d_{i}\left(f\left(x_{i}(t)\right)-f(s(t))\right)^{T}\left(h\left(x_{i}(t)\right)-h(s(t))\right) \\
& +c\left(\sum_{j=1}^{N} g_{i j}\left(h\left(x_{j}(t)\right)-h(s(t))\right)\right)^{T}\left(f\left(x_{i}(t)\right)-f(s(t))\right) \\
& +c^{2}\left(\sum_{j=1}^{N} g_{i j}\left(h\left(x_{j}(t)\right)-h(s(t))\right)\right)^{T} \sum_{j=1}^{N} g_{i j}\left(h\left(x_{j}(t)\right)\right. \\
& -h(s(t)))-c^{2} d_{i}\left(\sum_{j=1}^{N} g_{i j}\left(h\left(x_{j}(t)\right)-h(s(t))\right)\right)^{T} \\
& \cdot\left(h\left(x_{i}(t)\right)-h(s(t))\right)-c d_{i}\left(h\left(x_{i}(t)\right)-h(s(t))\right)^{T} \\
& \cdot\left(f\left(x_{i}(t)\right)-f(s(t))\right)-c^{2} d_{i}\left(h\left(x_{i}(t)\right)\right. \\
& -h(s(t)))^{T} \sum_{j=1}^{N} g_{i j}\left(h\left(x_{j}(t)\right)-h(s(t))\right)+c^{2} d_{i}^{2}\left(h\left(x_{i}(t)\right)\right.
\end{aligned}
$$$$
\begin{aligned}
& \left.\left.-h(s(t)))^{T}\left(h\left(x_{i}(t)\right)-h(s(t))\right)\right]\right\} \\
\leq & \sum_{i=1}^{N}\left\{2\left\|z_{i}(t)\right\|\left\|f\left(x_{i}(t)\right)-f(s(t))\right\|\right. \\
& +2 c \sum_{j=1}^{N} g_{i j} z_{\mathrm{i}}^{T}(t)\left(h\left(x_{j}(t)\right)-h(s(t))\right) \\
& -2 c d_{i} z_{i}^{T}(t)\left(h\left(x_{i}(t)\right)-h(s(t))\right) \\
& +\mu(t)\left[\left\|f\left(x_{i}(t)\right)-f(s(t))\right\|^{2}+c \sum_{j=1}^{N} g_{i j}\right. \\
& +\left(f\left(x_{i}(t)\right)-f(s(t))\right)^{T}\left(h\left(x_{j}(t)\right)-h(s(t))\right) \\
& -c d_{i}\left(f\left(x_{i}(t)\right)-f(s(t))\right)^{T}\left(h\left(x_{i}(t)\right)-h(s(t))\right) \\
& +c \sum_{j=1}^{N} g_{i j}\left(h\left(x_{j}(t)\right)-h(s(t))\right)^{T}\left(f\left(x_{i}(t)\right)-f(s(t))\right) \\
& +c^{2}\left(\sum_{j=1}^{N} g_{j i}\left(h\left(x_{j}(t)\right)-h(s(t))\right)^{T}\right) \\
& +\left(\sum_{j=1}^{N} g_{i j}\left(h\left(x_{j}(t)\right)-h(s(t))\right)\right) \\
& +c^{2} d_{i}\left(\sum_{j=1}^{N} g_{i j}\left(h\left(x_{j}(t)\right)-h(s(t))\right)\right)^{2}\left(h\left(x_{i}(t)\right)-h(s(t))\right)^{T}\left(h\left(x_{i}(t)\right)-h(s(t))\right) \\
& -c d_{\mathrm{i}}\left(h\left(x_{i}(t)\right)-h(s(t))\right)^{T}\left(f\left(x_{i}(t)\right)-f(s(t))\right) \\
& -c^{2} d_{i}\left(h\left(x_{i}(t)\right)-h(s(t))\right)^{T}\left(\sum_{j=1}^{N} g_{i j}\left(h\left(x_{j}(t)\right)-h(s(t))\right)\right)
\end{aligned}
$$

By assumption (A1), the following inequalities can be obtained:

$$
\begin{aligned}
V^{\Delta}(t) \leq & \sum_{i=1}^{N} 2 l_{1}\left\|z_{i}(t)\right\|^{2}+V_{1}(t)+V_{2}(t) \\
& +\mu(t)\left[\sum_{i=1}^{N} l_{1}^{2}\left\|z_{i}(t)\right\|^{2}+V_{3}(t)+V_{4}(t)\right. \\
& \left.+V_{5}(t)+V_{6}(t)+V_{7}(t)+V_{8}(t)\right]
\end{aligned}
$$

where $V_{1}(t)=2 c \sum_{i=1}^{N} \sum_{j=1}^{N} g_{i j} z_{i}^{T}(t)\left(h\left(x_{j}(t)\right)-h(s(t))\right), V_{2}(t)$ $=-2 c \sum_{i=1}^{N} d_{i} z_{i}^{T}(t)\left(h\left(x_{i}(t)\right)-h(s(t))\right), \quad V_{3}(t)=2 c \sum_{i=1}^{N} \sum_{j=1}^{N} g_{i j}$ $\left(f\left(x_{i}(t)\right)-f(s(t))\right)^{T}\left(h\left(x_{j}(t)\right)-h(s(t))\right), \quad V_{4}(t)=-2 c \sum_{i=1}^{N} d_{i}$ $\left(f\left(x_{i}(t)\right)-f(s(t))\right)^{T}\left(h\left(x_{i}(t)\right)-h(s(t))\right), V_{5}(t)=c^{2} \sum_{i=1}^{N}\left(\sum_{j=1}^{N}\right.$ $\left.g_{j i}\left(h\left(x_{j}(t)\right)-h(s(t))\right)^{T}\right)\left(\sum_{j=1}^{N} g_{i j}\left(h\left(x_{j}(t)\right)-h(s(t))\right)\right), V_{6}(t)=$ 
$-c^{2} \sum_{i=1}^{N} d_{i}\left(\sum_{j=1}^{N} g_{i j}\left(h\left(x_{j}(t)\right)-h(s(t))\right)\right)^{T}\left(h\left(x_{i}(t)\right)-h(s(t))\right)$, $V_{7}(t)=-c^{2} \sum_{i=1}^{N} d_{i}\left(h\left(x_{i}(t)\right)-h(s(t))\right)^{T}\left(\sum_{j=1}^{N} g_{i j}\left(h\left(x_{j}(t)\right)-h\right.\right.$ $(s(t))))$, and $V_{8}(t)=c^{2} \sum_{i=1}^{N} d_{i}^{2}\left(h\left(x_{i}(t)\right)-h(s(t))\right)^{T}\left(h\left(x_{i}(t)\right)\right.$ $-h(s(t)))$.

We introduce the notations

$z(t)=\left(z_{1}^{T}(t), z_{2}^{T}(t), \cdots, z_{N}^{T}(t)\right)^{T}$,

$H(z(t))=\left(\left(h\left(x_{1}(t)\right)-h(s(t))\right)^{T},\left(h\left(x_{2}(t)\right)\right.\right.$

$\left.-h(s(t)))^{T}, \cdots,\left(h\left(x_{N}(t)\right)-h(s(t))\right)^{T}\right)^{T}$,

$F(z(t))=\left(\left(f\left(x_{1}(t)\right)-f(s(t))\right)^{T},\left(f\left(x_{2}(t)\right)\right.\right.$

$$
\left.-f(s(t)))^{T}, \cdots,\left(f\left(x_{N}(t)\right)-f(s(t))\right)^{T}\right)^{T} .
$$

$$
\begin{aligned}
& \leq-2 c \sum_{1 \leq i<j \leq N} g_{i j}\left(x_{i}(t)-x_{j}(t)\right)^{T} K\left(x_{i}(t)-x_{j}(t)\right) \\
& =-2 c \sum_{1 \leq i<j \leq N} g_{i j}\left(z_{i}(t)\right)-z_{j}(t)^{T} K\left(z_{i}(t)-z_{j}(t)\right) \\
& =2 c z^{T}(t)(G \otimes K) z(t) .
\end{aligned}
$$

By assumption (A3), we get

$$
\begin{aligned}
V_{4}(t) & =-2 c \sum_{i=1}^{N} d_{i}\left(f\left(x_{i}(t)\right)-f(s(t))\right)^{T}\left(h\left(x_{i}(t)\right)-h(s(t))\right) \\
& \leq-2 c \sum_{i=1}^{N} d_{i} z_{i}^{T}(t) K z_{i}(t)=-2 c z^{T}(t)(D \otimes K) z(t) .
\end{aligned}
$$

By assumption (A2) and Lemma 15, we have

$$
\begin{aligned}
V_{1}(t)= & 2 c \sum_{i=1}^{N} \sum_{j=1}^{N} z_{i}^{T}(t) g_{i j}\left(h\left(x_{j}(t)\right)-h(s(t))\right) \\
= & 2 c z^{T}(t)\left(G \otimes I_{n}\right) H(z(t)) \\
= & -2 c \sum_{1 \leq i<j \leq N} g_{i j}\left(z_{i}(t)-z_{j}(t)\right)^{T}\left[\left(h\left(x_{i}(t)\right)-h(s(t))\right)\right. \\
& \left.-\left(h\left(x_{j}(t)\right)-h(s(t))\right)\right] \\
= & -2 c \sum_{1 \leq i<j \leq N} g_{i j}\left(x_{i}(t)-x_{j}(t)\right)^{T}\left[h\left(x_{i}(t)\right)-h\left(x_{j}(t)\right)\right] \\
\leq & -2 c l_{2} \sum_{1 \leq i<j \leq N} g_{i j}\left(x_{i}(t)-x_{j}(t)\right)^{T}\left(x_{i}(t)-x_{j}(t)\right) \\
= & -2 c l_{2} \sum_{1 \leq i<j \leq N} g_{i j}\left(z_{i}(t)-z_{j}(t)\right)^{T}\left(z_{i}(t)-z_{j}(t)\right) \\
= & \left.2 c l_{2} z^{T}(t)\left(G \otimes I_{n}\right) z(t)\right) .
\end{aligned}
$$

By assumption (A2), we get

$$
\begin{aligned}
V_{2}(t) & =-2 c \sum_{i=1}^{N} d_{i} z_{i}^{T}(t)\left(h\left(x_{i}(t)\right)-h(s(t))\right) \\
& \leq-2 c l_{2} \sum_{i=1}^{N} d_{i} z_{i}^{T}(t) z_{i}(t)=-2 c l_{2} z^{T}(t)\left(D \otimes I_{n}\right) z(t) .
\end{aligned}
$$

By assumption (A3) and Lemma 15, we have

$$
\begin{aligned}
V_{3}(t) & =2 c \sum_{i=1}^{N} \sum_{j=1}^{N} g_{i j}\left(f\left(x_{i}(t)\right)-f(s(t))\right)^{T}\left(h\left(x_{j}(t)\right)-h(s(t))\right) \\
& =2 c F^{T}(z(t))\left(G \otimes I_{n}\right) H(z(t)) \\
& =-2 c \sum_{1 \leq i<j \leq N} g_{i j}\left(f\left(x_{i}(t)\right)-f\left(x_{j}(t)\right)\right)^{T}\left(h\left(x_{i}(t)\right)-h\left(x_{j}(t)\right)\right)
\end{aligned}
$$

By calculating, one has

$$
\begin{aligned}
V_{5}(t)= & c^{2} \sum_{i=1}^{N}\left(\sum_{j=1}^{N} g_{j i}\left(h\left(x_{j}(t)\right)-h(s(t))\right)^{T}\right) \\
& \cdot\left(\sum_{j=1}^{N} g_{i j}\left(h\left(x_{j}(t)\right)-h(s(t))\right)\right) \\
= & c^{2} H^{T}(z(t))\left(G^{2} \otimes I_{n}\right) H(z(t)), \\
V_{6}(t)= & -c^{2} \sum_{i=1}^{N} d_{i}\left(\sum_{j=1}^{N} g_{j i}\left(h\left(x_{j}(t)\right)-h(s(t))\right)^{T}\right) \\
& \cdot\left(h\left(x_{i}(t)\right)-h(s(t))\right) \\
= & -c^{2} H^{T}(z(t))\left(G D \otimes I_{n}\right) H(z(t)), \\
V_{7}(t)= & -c^{2} \sum_{i=1}^{N} d_{i}\left(h\left(x_{i}(t)\right)-h(s(t))\right)^{T} \\
& \cdot\left(\sum_{j=1}^{N} g_{i j}\left(h\left(x_{j}(t)\right)-h(s(t))\right)\right) \\
= & -c^{2} H^{T}(z(t))\left(D G \otimes I_{n}\right) H(z(t)), \\
V_{8}(t)= & c^{2} \sum_{i=1}^{N} d_{i}^{2}\left(h\left(x_{i}(t)\right)-h(s(t))\right)^{T} \\
& \cdot\left(h\left(x_{i}(t)\right)-h(s(t))\right) \\
= & c^{2} H^{T}(z(t))\left(D^{2} \otimes I_{n}\right) H(z(t)) .
\end{aligned}
$$

Hence, by Lemmas 14((1), (2), (4), (5)) and 17 (4) and assumption (A4), we have

$$
\begin{aligned}
& V_{5}(t)+V_{6}(t)+V_{7}(t)+V_{8}(t) \\
& \left.\quad=c^{2} H^{T}(z(t))\left[\left(G^{2}-G D-D G+D^{2}\right) \otimes I_{n}\right)\right] H(z(t)) \\
& \quad=c^{2} H^{T}(z(t))\left((G-D)^{2} \otimes I_{n}\right) H(z(t)) \\
& \quad \leq c^{2} \lambda_{\max }\left((G-D)^{2}\right) H^{T}(z(t)) H(z(t))
\end{aligned}
$$




$$
\begin{aligned}
& =c^{2} \lambda_{\max }\left((G-D)^{2}\right) \sum_{i=1}^{N}\left(h\left(x_{i}(t)\right)-h(s(t))\right)^{T}\left(h\left(x_{i}(t)\right)\right. \\
& -h(s(t)))=c^{2} \lambda_{\max }\left((G-D)^{2}\right) \sum_{i=1}^{N}\left\|h\left(x_{i}(t)\right)-h(s(t))\right\|^{2} \\
& \leq c^{2} l_{3}^{2} \lambda_{\max }\left((G-D)^{2}\right) \sum_{i=1}^{N}\left\|z_{i}(t)\right\|^{2} .
\end{aligned}
$$

In view of (22), (24), (25), (26), (27), (29), and Lemma $14((1),(2))$, one can get

$$
\begin{aligned}
V^{\Delta}(t) \leq & 2 l_{1} z^{T}(t) z(t)+2 c l_{2} z^{T}(t)\left[(\mathrm{G}-\mathrm{D}) \otimes I_{n}\right] z(t) \\
& +\mu(t)\left\{l_{1}^{2} z^{T}(t) z(t)+2 c z^{T}(t)[(G-D) \otimes K] z(t)\right. \\
& \left.+c^{2} l_{3}^{2} \lambda_{\max }\left((G-D)^{2}\right) z^{T}(t) z(t)\right\} \\
= & z^{T}(t)\left\{2 l_{1} I_{n N}+2 c l_{2}\left[(G-D) \otimes I_{n}\right]\right. \\
& +\mu(t)\left[l_{1}^{2} I_{n N}+2 c((G-D) \otimes K)\right. \\
& \left.\left.+c^{2} l_{3}^{2} \lambda_{\max }\left((G-D)^{2}\right) I_{n N}\right]\right\} z(t) .
\end{aligned}
$$

From (20), we have

$$
V^{\Delta}(t) \leq \theta(t) V(t), \quad t \in[0,+\infty)_{\mathbb{T}} .
$$

By Lemma 10, it implies that

$$
V(t) \leq V(0) e_{\theta}(t, 0), \quad t \in[0,+\infty)_{\mathbb{T}} .
$$

Since $\theta \in \mathscr{R}^{+}(\mathbb{T} ; \mathbb{R})$ and $\lim _{t \rightarrow \infty} e_{\theta}(t, 0)=0, V(t) \longrightarrow 0$ as $t \longrightarrow \infty$. Thus, the network (12) is synchronized by pinning control.

Theorem 20. Let $\mu(t) \leq \mu^{*}$ for all $t \in \mathbb{T}$. Suppose that assumptions (A1), (A2), (A3), and (A4) hold. Then, the network (12) is synchronized by pinning control, if there exists a constant $\alpha<0$ with $\alpha \in \mathscr{R}^{+}(\mathbb{T} ; \mathbb{R})$ such that

$$
\begin{aligned}
& 2 l_{1} I_{n N}+2 c l_{2}\left[(G-D) \otimes I_{n}\right]+\mu^{*}\left[l_{1}^{2} I_{n N}+2 c((G-D) \otimes K)\right. \\
& \left.\quad+c^{2} l_{3}^{2} \lambda_{\max }\left((G-D)^{2}\right) I_{n N}\right] \leq \alpha I_{n N}
\end{aligned}
$$

holds.

Proof. Lemma 11 yields $\lim _{t \rightarrow \infty} e_{\alpha}(t, 0)=0$. Choose the Lyapunov function $V(t)=\sum_{i=1}^{N} z_{i}^{T}(t) z_{i}(t), \quad t \in[0,+\infty)_{\mathbb{T}}$. Note that

$$
\begin{aligned}
V^{\Delta}(t)= & \sum_{i=1}^{N}\left[2 z_{i}^{T}(t) z_{i}^{\Delta}(t)+\mu(t)\left(z_{i}^{\Delta}(t)\right)^{T} z_{i}^{\Delta}(t)\right] \\
& \leq \sum_{i=1}^{N}\left[2 z_{i}^{T}(t) z_{i}^{\Delta}(t)+\mu^{*}\left(z_{i}^{\Delta}(t)\right)^{T} z_{i}^{\Delta}(t)\right] .
\end{aligned}
$$

The rest proof of the theorem is analogy with the proof of Theorem 19.

Theorem 21. Let $\mu(t) \leq \mu^{*}$ for all $t \in \mathbb{T}$. Suppose that assumptions (A1), (A2), (A3), and (A4) hold, and $K$ is symmetric in assumption (A3). Then, the network (12) is synchronized by pinning control, if $\lambda=\lambda_{\max }\left(2 l_{1} I_{n N}+2 c l_{2}\left[(G-D) \otimes I_{n}\right]+\mu^{*}[\right.$ $\left.\left.l_{1}^{2} I_{n N}+2 c((G-D) \otimes K)+c^{2} l_{3}^{2} \lambda_{\max }\left((G-D)^{2}\right) I_{n N}\right]\right)<0$ and $\lambda$ $\in \mathscr{R}^{+}(\mathbb{T} ; \mathbb{R})$.

Proof. Choose the Lyapunov function $V(t)=\sum_{i=1}^{N} z_{i}^{T}(t) z_{i}(t)$, $t \in[0,+\infty)_{\mathbb{T}}$. Taking into account the inequality (34), by analogy with the proof of Theorem 19, one has

$$
\begin{aligned}
V^{\Delta}(t) \leq & z^{T}(t)\left\{2 l_{1} I_{n N}+2 c l_{2}\left[(G-D) \otimes I_{n}\right]+\mu^{*}\left[l_{1}^{2} I_{n N}\right.\right. \\
& \left.\left.+2 c((G-D) \otimes K)+c^{2} l_{3}^{2} \lambda_{\max }\left((G-D)^{2}\right) I_{n N}\right]\right\} z(t) .
\end{aligned}
$$

Hence, by Lemma 17(4), the following estimate holds:

$$
\begin{aligned}
& V^{\Delta}(t) \leq \lambda_{\max }\left(2 l_{1} I_{n N}+2 c l_{2}\left[(G-D) \otimes I_{n}\right]+\mu^{*}\left[l_{1}^{2} I_{n N}\right.\right. \\
& \left.\left.\quad+2 c((G-D) \otimes K)+c^{2} l_{3}^{2} \lambda_{\max }\left((G-D)^{2}\right) I_{n N}\right]\right) z^{T}(t) z(t) \\
& \quad=\lambda z^{T}(t) z(t)=\lambda V(t), \quad t \in[0,+\infty)_{\mathbb{T}} .
\end{aligned}
$$

By Lemma 10 , we have $V(t) \leq V(0) e_{\lambda}(t, 0), \quad t \in$ $[0,+\infty)_{\mathbb{T}}$. Since $\lambda<0$ and $\lambda \in \mathscr{R}^{+}(\mathbb{T} ; \mathbb{R})$, it follows from Lemma 11 that $\lim _{t \rightarrow \infty} e_{\lambda}(t, 0)=0$. Therefore, $V(t) \longrightarrow 0$ as $t$ $\longrightarrow \infty$. This completes the proof.

Corollary 22. Let $\mu(t) \leq \mu^{*}$ for all $t \in \mathbb{T}$. Suppose that assumptions (A1), (A2), (A3), and (A4) hold, and $K$ is symmetric in assumption (A3). If inequality $2 l_{1}+2 c l_{2} \lambda_{\max }(G-D)+$ $\mu^{*}\left[l_{1}^{2}+2 c \lambda_{\max }((G-D) \otimes K)+c^{2} l_{3}^{2} \lambda_{\max }\left((G-D)^{2}\right)\right]<0$ holds, and $2 l_{1}+2 c l_{2} \lambda_{\max }(G-D)+\mu^{*}\left[l_{1}^{2}+2 c \lambda_{\max }((G-D) \otimes K)+\right.$ $\left.c^{2} l_{3}^{2} \lambda_{\max }\left((G-D)^{2}\right)\right] \in \mathscr{R}^{+}(\mathbb{T} ; \mathbb{R})$, then the network (12) is synchronized by pinning control.

Proof. By Lemmas 14((4), (5)) and 17((5)) and Theorem 21, it is not hard to see the result holds when $\mu^{*}>0$ and $\mu^{*}=0$.

Next, let us consider the particular case when the function $h(x)$ is linear: $h(x)=\Gamma x$, where $\Gamma \in \mathbb{R}^{n \times n}$. The network (11) can be written as

$$
\begin{aligned}
& x_{i}^{\Delta}(t)=f\left(x_{i}(t)\right)+c \sum_{j=1}^{N} g_{i j} \Gamma x_{j}(t), \quad t \in[0,+\infty)_{\mathbb{T}}, \\
& \quad i=1,2, \cdots, N .
\end{aligned}
$$


We write the network (12) as the following

$$
\begin{aligned}
& x_{i}^{\Delta}(t)=f\left(x_{i}(t)\right)+c \sum_{j=1}^{N} g_{i j} \Gamma x_{j}(t)+u_{i}(t), \quad t \in[0,+\infty)_{\mathbb{T}}, \\
& \quad i=1,2, \cdots, N
\end{aligned}
$$

where $u_{i}(t)=-c d_{i} \Gamma\left(x_{i}(t)-s(t)\right), \quad t \in[0,+\infty)_{\mathbb{T}}, \quad d_{i}=d>0$, $(i=1,2, \cdots, l), \quad d_{i}=0, \quad(i=l+1, l+2, \cdots, N)$, and $s(t)$ is a solution of system: $s^{\Delta}(t)=f(s(t)), \quad t \in[0,+\infty)_{\mathbb{T}}$. Meanwhile, the assumption (A3) can be described as

$\left(\mathrm{A} 3^{\prime}\right)$. There exists a constant matrix $\bar{K} \in \mathbb{R}^{n \times n}$, such that

$$
\begin{aligned}
& (x-y)^{T} \Gamma^{T}(f(x)-f(y)) \\
& \geq(x-y)^{T} \bar{K}(x-y), \quad \text { for any } x, y \in \mathbb{R}^{n} .
\end{aligned}
$$

Corollary 23. Let $\mu(t) \leq \mu^{*}$ for all $t \in \mathbb{T}$ and $\Gamma$ be symmetric positive definite. Suppose that assumptions (A1) and (A3') hold. The network (38) is synchronized by pinning control if there exists a constant $\beta<0$ with $\beta \in \mathscr{R}^{+}(\mathbb{T} ; \mathbb{R})$ such that the following inequality is satisfied:

$$
\begin{aligned}
& 2 l_{1} I_{n N}+2 c \lambda_{\min }(\Gamma)\left[(G-D) \otimes I_{n}\right]+\mu^{*}\left[l_{1}^{2} I_{n N}+2 c((G-D) \otimes \bar{K})\right. \\
& \left.\quad+c^{2}\left(\lambda_{\max }(\Gamma)\right)^{2} \lambda_{\max }\left((G-D)^{2}\right) I_{n N}\right] \leq \beta I_{n N} .
\end{aligned}
$$

Proof. Since $\Gamma$ is symmetric positive definite, one has $0<$ $\lambda_{\text {min }}(\Gamma)$. By Lemma $17(3), \Gamma^{2}$ is also symmetric positive definite. From Lemma $17((4),(6))$, it is easy to see that

$$
\begin{gathered}
(x-y)^{T} \Gamma(x-y) \geq \lambda_{\min }(\Gamma)(x-y)^{T}(x-y), \quad \text { for any } x, y \in \mathbb{R}^{n}, \\
\|\Gamma(x-y)\| \leq \lambda_{\max }(\Gamma)\|x-y\|, \quad \text { for any } x, y \in \mathbb{R}^{n} .
\end{gathered}
$$

That is, assumptions (A2) and (A4) hold, where $l_{2}=$ $\lambda_{\min }(\Gamma)$ and $l_{3}=\lambda_{\max }(\Gamma)$. Now, the result follows from Theorem 20 , immediately.

By Theorem 21 and Corollary 23, we have the following corollary.

Corollary 24. Let $\mu(t) \leq \mu^{*}$ for all $t \in \mathbb{T}$ and $\Gamma$ be symmetric positive definite. Suppose that assumptions (A1) and (A3') hold, and $\bar{K}$ is symmetric in assumption $\left(A 3^{\prime}\right)$. The network (38) is synchronized by pinning control if $\gamma=\lambda_{\max }\left(2 l_{1} I_{n N}+2\right.$ $c \lambda_{\min }(\Gamma)\left[(G-D) \otimes I_{n}\right]+\mu^{*}\left[l_{1}^{2} I_{n N}+2 c((G-D) \otimes \bar{K})+c^{2}\right.$ $\left.\left.\left(\lambda_{\max }(\Gamma)\right)^{2} \lambda_{\max }\left((G-D)^{2}\right) I_{n N}\right]\right)<0$ and $\gamma \in \mathscr{R}^{+}(\mathbb{T} ; \mathbb{R})$.

Obviously, the research of synchronization problem for nonlinearly coupled complex dynamical networks on time scales is more general. It contains continuous-time and discrete-time nonlinearly coupled complex dynamical networks. In addition, it contains linearly coupled complex dynamical networks on time scales.

Remark 25. According to the previous works [12, 26, 31, 35, $39,40]$, the synchronization problem of complex dynamical networks with delay on time scales by pinning control strategy can be investigated, but there are some challenges for the effects of time delays and the various forms of time scales.

\section{Numerical Examples}

In this section, two numerical examples are given to verify the theoretical results established above.

Example 1. Consider 2-dimensional nonlinearly coupled complete network with ten nodes on time scale $\mathbb{T}$, which is described by

$$
\begin{aligned}
& x_{i}^{\Delta}(t)=f\left(x_{i}(t)\right)+c \sum_{j=1}^{10} g_{i j} h\left(x_{j}(t)\right), \quad t \in[0,+\infty)_{\mathbb{T}}, \\
& i=1,2, \cdots, 10,
\end{aligned}
$$

where $\mathbb{T}=\bigcup_{k=0}^{\infty}[k, k+0.7], \quad k \in \mathbb{N} \cup\{0\}$, in this case the graininess function of $\mathbb{T}$ is given by

$$
\mu(t)= \begin{cases}0, & t \in \bigcup_{k=0}^{\infty}[k, k+0.7) \\ 0.3, & t=k+0.7\end{cases}
$$

$$
\begin{aligned}
& \quad x_{i}(t)=\left(x_{i 1}(t), x_{i 2}(t)\right)^{T},(i=1,2, \cdots, 10), \quad f\left(x_{i}(t)\right)= \\
& \left(f_{1}\left(x_{i 1}(t)\right), f_{2}\left(x_{i 2}(t)\right)\right)^{T} \text { with } f_{j}\left(x_{i j}(t)\right)=0.01\left[\tanh \left(x_{i j}(t)\right)+\right. \\
& \left.x_{i j}(t)\right],(j=1,2), h\left(x_{i}(t)\right)=\left(h_{1}\left(x_{i 1}(t)\right), h_{2}\left(x_{i 2}(t)\right)\right)^{T} \text { with } h_{j} \\
& \left(x_{i j}(t)\right)=0.01 \tanh \left(x_{i j}(t)\right)+0.1 x_{i j}(t),(j=1,2), c=0.2, \text { and } \\
& \text { the corresponding matrix } G \text { is }
\end{aligned}
$$

$$
G=\left(\begin{array}{cccccccccc}
-9 & 1 & 1 & 1 & 1 & 1 & 1 & 1 & 1 & 1 \\
1 & -9 & 1 & 1 & 1 & 1 & 1 & 1 & 1 & 1 \\
1 & 1 & -9 & 1 & 1 & 1 & 1 & 1 & 1 & 1 \\
1 & 1 & 1 & -9 & 1 & 1 & 1 & 1 & 1 & 1 \\
1 & 1 & 1 & 1 & -9 & 1 & 1 & 1 & 1 & 1 \\
1 & 1 & 1 & 1 & 1 & -9 & 1 & 1 & 1 & 1 \\
1 & 1 & 1 & 1 & 1 & 1 & -9 & 1 & 1 & 1 \\
1 & 1 & 1 & 1 & 1 & 1 & 1 & -9 & 1 & 1 \\
1 & 1 & 1 & 1 & 1 & 1 & 1 & 1 & -9 & 1 \\
1 & 1 & 1 & 1 & 1 & 1 & 1 & 1 & 1 & -9
\end{array}\right) .
$$



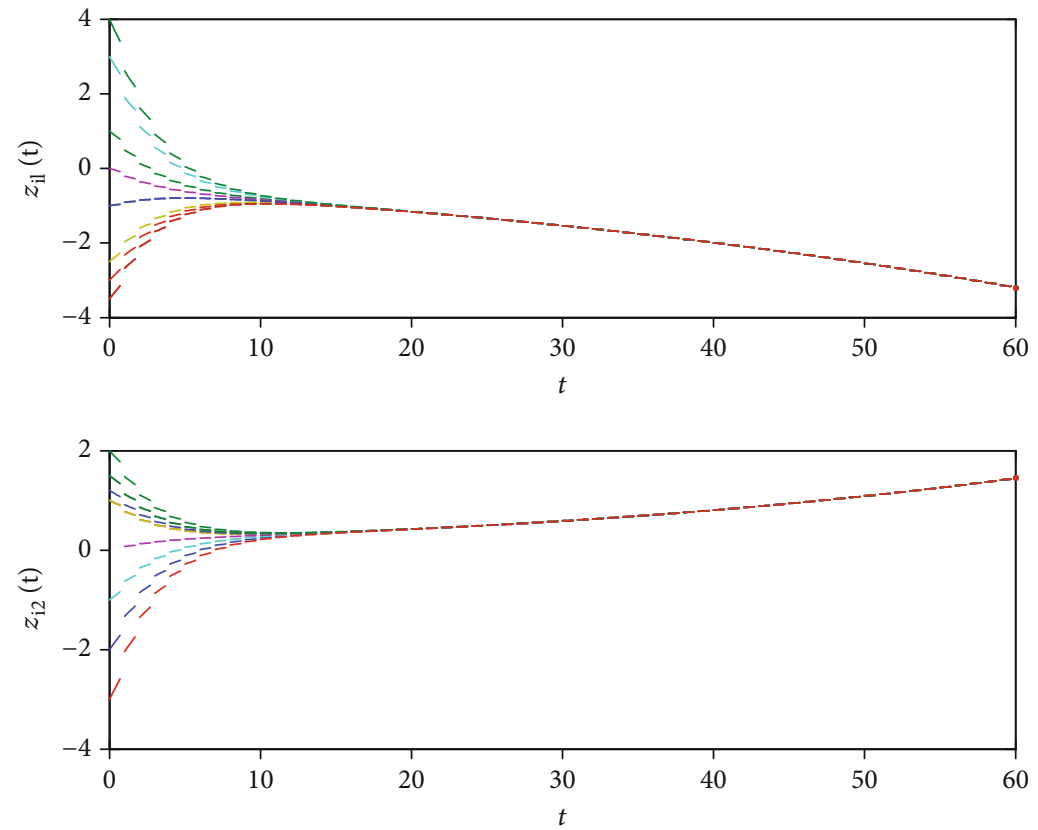

FIgURE 1: Error $z_{i 1}$ and $z_{i 2}$ of the system (42) without control.
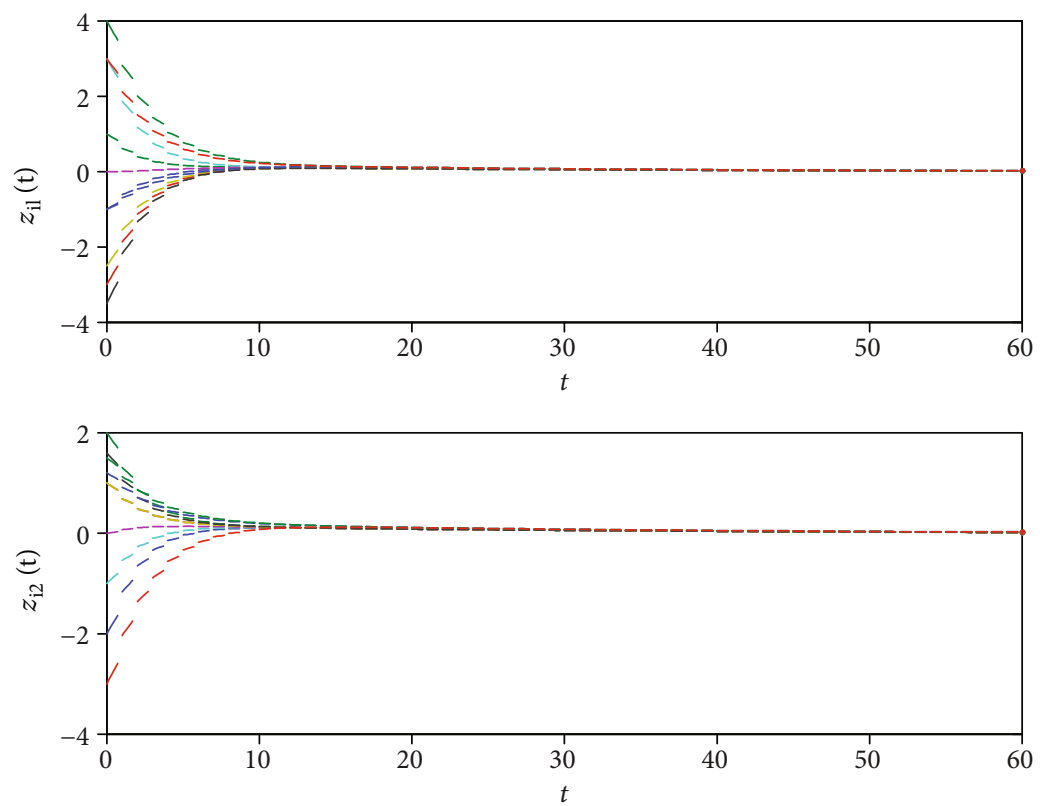

FIGURE 2: Synchronization error $z_{i 1}$ and $z_{i 2}$ of the system (42).

Note that each isolated node of network (42) is a 2dimensional nonlinear system described by

$$
\begin{aligned}
s^{\Delta}(t) & =\left(\begin{array}{c}
s_{1}^{\Delta}(t) \\
s_{2}^{\Delta}(t)
\end{array}\right) \\
& =\left(\begin{array}{c}
0.01\left[\tanh \left(s_{1}(t)\right)+s_{1}(t)\right] \\
0.01\left[\tanh \left(s_{2}(t)\right)+s_{2}(t)\right]
\end{array}\right), \quad t \in[0,+\infty)_{\mathbb{T}} .
\end{aligned}
$$

It is not difficult to obtain that $f(\cdot)$ and $h(\cdot)$ satisfy assumptions (A1), (A2), (A3), and (A4) with $l_{1}=0.02, l_{2}=$ $0.1, l_{3}=0.11$, and constant matrix

$$
K=\left(\begin{array}{cc}
0.001 & 0 \\
0 & 0.001
\end{array}\right)
$$

The objective here is to synchronize the network (42) to the solution $s=0$ (2-dimensional zero vector) of (45) by pinning control. From Figure 1, we see the complex 

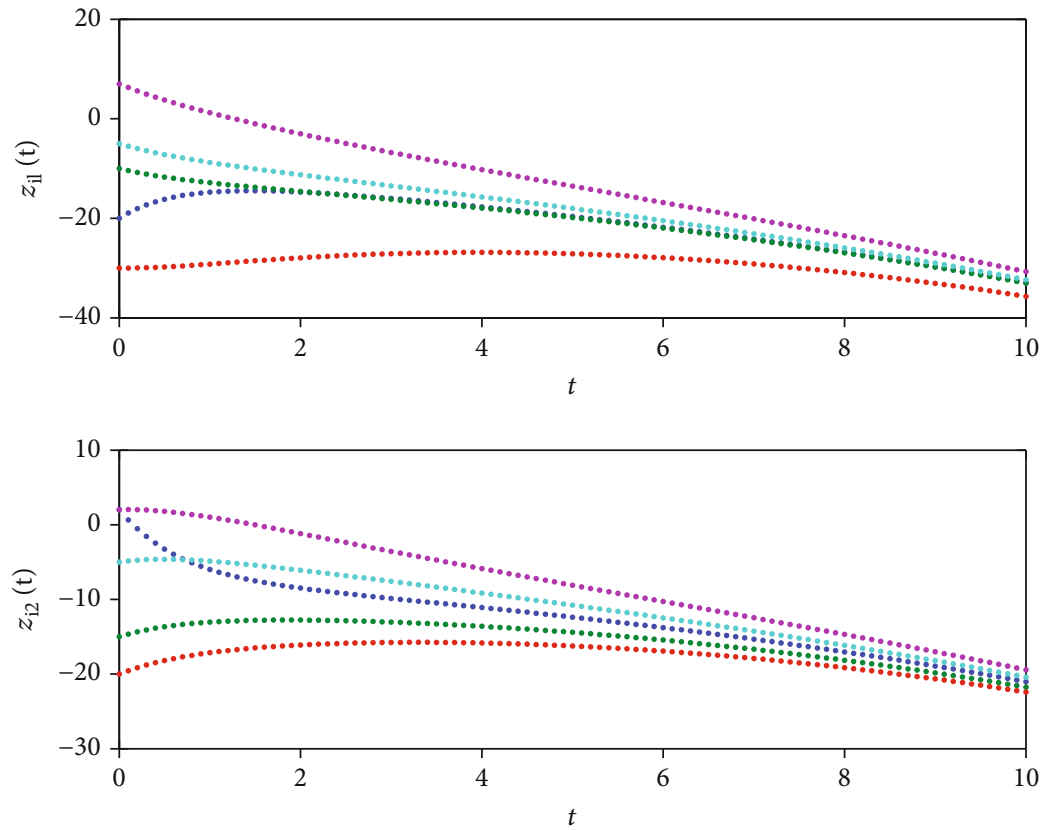

FIgURE 3: Error $z_{i 1}$ and $z_{i 2}$ of the system (47) without control.
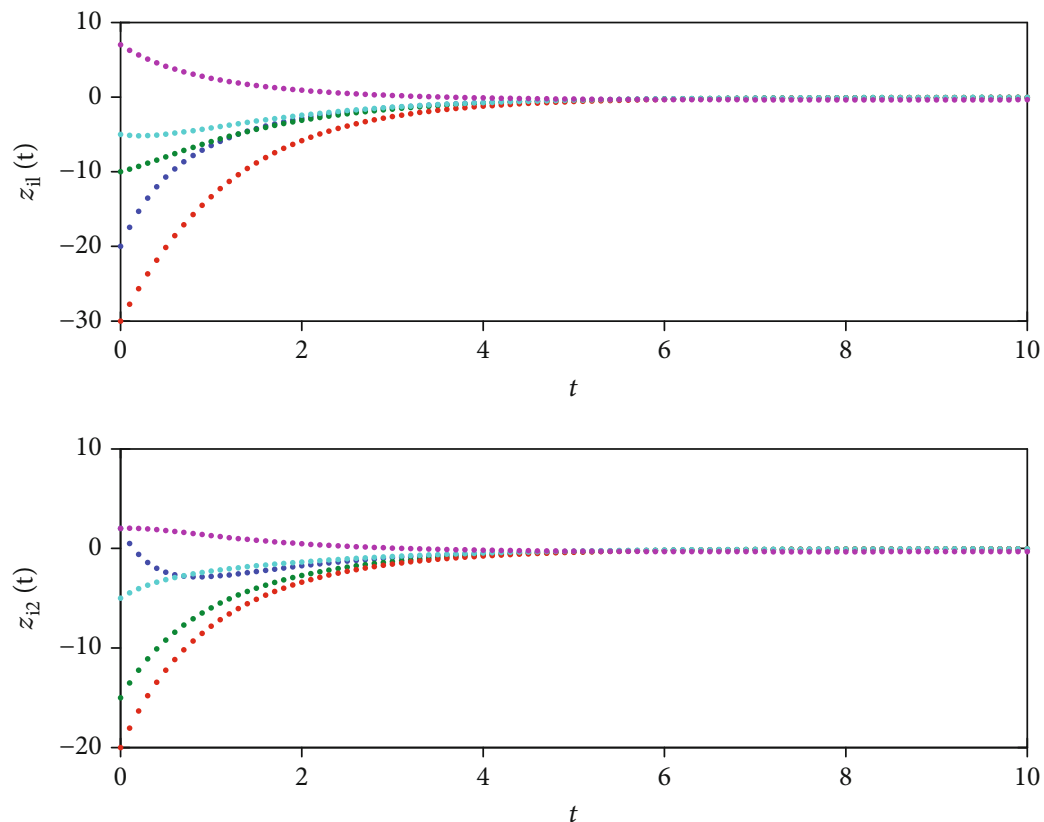

FIGURE 4: Synchronization error $z_{i 1}$ and $z_{i 2}$ of the system (47).

dynamical network (42) without control cannot reach synchronization with $s=0$. Now, we apply pinning control to the network (42) with matrix $D=\operatorname{diag}(3,3,3,3,3,3,3,0$, $0,0)$. By calculations, all conditions of Theorem 21 are fulfilled. Then by Theorem 21, the network (42) can achieve synchronization under the above pinning control strategy. Figure 2 also shows that the synchronization is realized.

Example 2. Consider 2-dimensional nonlinearly star-shaped network with five nodes on time scale $\mathbb{T}$, which is described by

$$
\begin{aligned}
x_{i}^{\Delta}(t)=f\left(x_{i}(t)\right)+c \sum_{j=1}^{5} g_{i j} h\left(x_{j}(t)\right), \\
\quad t \in[0,+\infty)_{\mathbb{T}}, i=1,2, \cdots, 5,
\end{aligned}
$$

where $\mathbb{T}=0.1 \mathbb{Z}$; in this case, the graininess function is $\mu(t)=0.1, x_{i}(t)=\left(x_{i 1}(t), x_{i 2}(t)\right)^{T}, \quad(i=1,2, \cdots, 5), f\left(x_{i}(t)\right)$ $=\left(f_{1}\left(x_{i 1}(t)\right), f_{2}\left(x_{i 2}(t)\right)\right)^{T}$ with $f_{j}\left(x_{i j}(t)\right)=0.01\left[\tanh \left(x_{i j}\right.\right.$ $\left.(t))+x_{i j}(t)\right],(j=1,2), \quad h\left(x_{i}(t)\right)=\Gamma x_{i}(t) \quad$ with $\quad \Gamma=\operatorname{diag}$ $(0.05,0.05), c=0.6$, and the corresponding matrix $G$ is 


$$
G=\left(\begin{array}{ccccc}
-4 & 1 & 1 & 1 & 1 \\
1 & -1 & 0 & 0 & 0 \\
1 & 0 & -1 & 0 & 0 \\
1 & 0 & 0 & -1 & 0 \\
1 & 0 & 0 & 0 & -1
\end{array}\right) .
$$

Note that each isolated node of network (47) is a 2dimensional nonlinear system described by

$$
\begin{aligned}
s^{\Delta}(t) & =\left(\begin{array}{c}
s_{1}^{\Delta}(t) \\
s_{2}^{\Delta}(t)
\end{array}\right) \\
& =\left(\begin{array}{l}
0.01\left[\tanh \left(s_{1}(t)\right)+s_{1}(t)\right] \\
0.01\left[\tanh \left(s_{2}(t)\right)+s_{2}(t)\right]
\end{array}\right), \quad t \in[0,+\infty)_{T} .
\end{aligned}
$$

$\Gamma$ be symmetric positive definite. It is not difficult to obtain that assumptions (A1) and $\left(\mathrm{A} 3^{\prime}\right)$ are satisfied with $l_{1}=0.02$ and constant matrix

$$
\bar{K}=\left(\begin{array}{cc}
0.0005 & 0 \\
0 & 0.0005
\end{array}\right) .
$$

The objective here is to synchronize the network (47) to the solution $s=0$ (2-dimensional zero vector) of (49) by pinning control. From Figure 3, we see the complex dynamical network (47) without control cannot reach synchronization with $s=0$. Now, we apply pinning control to the network (47) with matrix $D=\operatorname{diag}(2.5,2.5,2.5,2.5,0)$. By calculations, all conditions of Corollary 24 are fulfilled. By Corollary 24, the network (47) can achieve synchronization under the above pinning control strategy. Figure 4 also shows that the synchronization is realized.

\section{Conclusions}

In this paper, we have investigated the synchronization problem of nonlinearly coupled complex dynamical networks on time scales by pinning control strategy. Some pinning synchronization criteria have been established which guarantee that the nonlinearly coupled complex dynamical networks on time scales can be pinned to some desired state. Two numerical examples have been given to verify the effectiveness of the obtained results.

\section{Data Availability}

The data used to support the findings of this study are included within the article.

\section{Conflicts of Interest}

The author declares that there is no conflict of interests regarding the publication of this paper.

\section{Acknowledgments}

This study is supported by the National Natural Science Foundation of China (11761047).

\section{References}

[1] S. H. Strogatz, "Exploring complex networks," Nature, vol. 410, no. 6825, pp. 268-276, 2001.

[2] X. F. Wang, "Complex networks: topology, dynamics and synchronization," International Journal of Bifurcation and Chaos, vol. 12, no. 5, pp. 885-916, 2002.

[3] G. Chen, X. F. Wang, and X. Li, Introduction to complex network: models, structures and dynamics, Higher Education Press, Beijing, 2012.

[4] M. Syed Ali and P. Balasubramaniam, "Stability analysis of uncertain fuzzy Hopfield neural networks with time delays," Communications in Nonlinear Science and Numerical Simulation, vol. 14, no. 6, pp. 2776-2783, 2009.

[5] M. Syed Ali, R. Vadivel, and R. Saravanakumar, "Event-triggered state estimation for Markovian jumping impulsive neural networks with interval time-varying delays," International Journal of Control, vol. 92, no. 2, pp. 270-290, 2017.

[6] R. Saravanakumar, M. Syed Ali, C. K. Ahn, H. R. Karimi, and P. Shi, "Stability of Markovian jump generalized neural networks with interval time-varying delays," IEEE Transactions on Neural Networks and Learning Systems, vol. 28, no. 8, pp. 1840-1850, 2017.

[7] M. Syed Ali, S. Saravanan, and J. Cao, "Finite-time boundedness, L2-gain analysis and control of Markovian jump switched neural networks with additive time-varying delays," Nonlinear Analysis: Hybrid Systems, vol. 23, pp. 27-43, 2017.

[8] M. Syed Ali, M. Usha, Z. Orman, and S. Arik, "Improved result on state estimation for complex dynamical networks with time varying delays and stochastic sampling via sampled-data control," Neural Networks, vol. 114, pp. 28-37, 2019.

[9] C. W. Wu, Synchronization in Coupled Chaotic Circuits and Systems, World Scientific, Singapore, 2002.

[10] C. W. Wu, Synchronization in Complex Networks of Nonlinear Dynamical Systems, World Scientific, Singapore, 2007.

[11] W. Lu and T. Chen, "Synchronization analysis of linearly coupled networks of discrete time systems," Physica D, vol. 198, no. 1-2, pp. 148-168, 2004.

[12] C. Li and G. Chen, "Synchronization in general complex dynamical networks with coupling delays," Physica A, vol. 343, pp. 263-278, 2004.

[13] Z. Li and G. Chen, "Global synchronization and asymptotic stability of complex dynamical networks," IEEE Transactions on Circuits and Systems-II: Express Briefs, vol. 53, no. 1, pp. 28-33, 2006.

[14] C. P. Li, W. G. Sun, and J. Kurths, "Synchronization of complex dynamical networks with time delays," Physica A, vol. 361, no. 1, pp. 24-34, 2006.

[15] X. Li and G. Chen, "Synchronization and desynchronization of complex dynamical networks: an engineering viewpoint," IEEE Transactions on Circuits and Systems-I: Fundamental Theory and Applications, vol. 50, no. 11, pp. 1381-1390, 2003.

[16] X. Liu and T. Chen, "Synchronization analysis for nonlinearlycoupled complex networks with an asymmetrical coupling matrix," Physica A, vol. 387, no. 16-17, pp. 4429-4439, 2008. 
[17] J. Zhou, J. Lu, and J. Lü, "Pinning adaptive synchronization of a general complex dynamical network," Automatica, vol. 44, no. 4, pp. 996-1003, 2008.

[18] J. Lu, J. Kurths, J. Cao, N. Mahdavi, and C. Huang, "Synchronization control for nonlinear stochastic dynamical networks: pinning impulsive strategy," IEEE Transactions on Neural Networks and Learning Systems, vol. 23, no. 2, pp. 285-292, 2012.

[19] W. Yu, G. Chen, J. Lü, and J. Kurths, "Synchronization via pinning control on general complex networks," SIAM Journal on Control and Optimization, vol. 51, no. 2, pp. 1395-1416, 2013.

[20] X. F. Wang and G. Chen, "Pinning control of scale-free dynamical networks," Physica A, vol. 310, no. 3-4, pp. 521$531,2002$.

[21] X. Li, X. Wang, and G. Chen, "Pinning a complex dynamical network to its equilibrium," IEEE Transactions on Circuits and Systems-I: Regular Papers, vol. 51, no. 10, pp. 2074-2087, 2004.

[22] T. Chen, X. Liu, and W. Lu, "Pinning complex networks by a single controller," IEEE Transactions on Circuits and Systems-I: Regular Papers, vol. 54, no. 6, pp. 1317-1326, 2007.

[23] W. Yu, G. Chen, and J. Lü, "On pinning synchronization of complex dynamical networks," Automatica, vol. 45, no. 2, pp. 429-435, 2009.

[24] Q. Song and J. Cao, "On pinning synchronization of directed and undirected complex dynamical networks," IEEE Transactions on Circuits and Systems-I: Regular Papers, vol. 57, no. 3, pp. 672-680, 2010.

[25] G. Chen, "Pinning control and synchronization on complex dynamical networks," International Journal of Control, Automation, and Systems, vol. 12, no. 2, pp. 221-230, 2014.

[26] Z. X. Liu, Z. Q. Chen, and Z. Z. Yuan, "Pinning control of weighted general complex dynamical networks with time delay," Physica A, vol. 375, no. 1, pp. 345-354, 2007.

[27] H. Su and X. Wang, Pinning Control of Complex Networked Systems: Synchronization, Consensus and Flocking of Networked Systems Via Pinning, Springer, Berlin, Heidelberg, 2013.

[28] X. Wang and H. Su, "Pinning control of complex networked systems: a decade after and beyond," Annual Reviews in Control, vol. 38, no. 1, pp. 103-111, 2014.

[29] H. Zhang, K. Li, and X. Fu, "On pinning control of some typical discrete-time dynamical networks," Communications in Nonlinear Science and Numerical Simulation, vol. 15, no. 2, pp. 182-188, 2010.

[30] W. Guo, F. Austin, and S. Chen, "Global synchronization of nonlinearly coupled complex networks with non-delayed and delayed coupling," Communications in Nonlinear Science and Numerical Simulation, vol. 15, no. 6, pp. 1631-1639, 2010.

[31] R. Cheng, M. Peng, and J. Zuo, "Pinning synchronization of discrete dynamical networks with delay coupling," Physica A, vol. 450, pp. 444-453, 2016.

[32] X. Liu and T. Chen, "Synchronization of nonlinear coupled networks via aperiodically intermittent pinning control," IEEE Transactions on Neural Networks and Learning Systems, vol. 26, no. 1, pp. 113-126, 2015.

[33] L. Y. Xiang, Z. X. Liu, Z. Q. Chen, F. Chen, and Z. Z. Yuan, "Pinning control of complex dynamical networks with general topology," Physica A, vol. 379, no. 1, pp. 298-306, 2007.

[34] Y. Li, J. Lou, Z. Wang, and F. E. Alsaadi, "Synchronization of dynamical networks with nonlinearly coupling function under hybrid pinning impulsive controllers," Journal of the Franklin Institute, vol. 355, no. 14, pp. 6520-6530, 2018.

[35] Q. Cheng and J. Cao, "Synchronization of complex dynamical networks with discrete time delays on time scales," Neurocomputing, vol. 151, pp. 729-736, 2015.

[36] X. Lu, Y. Wang, and Y. Zhao, "Synchronization of complex dynamical networks on time scales via Wirtinger-based inequality," Neurocomputing, vol. 216, pp. 143-149, 2016.

[37] A. Ogulenko, "Asymptotical properties of social network dynamics on time scales," Journal of Computational and Applied Mathematics, vol. 319, pp. 413-422, 2017.

[38] X. Liu and K. Zhang, "Synchronization of linear dynamical networks on time scales: pinning control via delayed impulses," Automatica, vol. 72, pp. 147-152, 2016.

[39] M. Syed Ali and J. Yogambigai, "Synchronization of complex dynamical networks with hybrid coupling delays on time scales by handling multitude Kronecker product terms," Applied Mathematics and Computation, vol. 291, pp. 244258, 2016.

[40] M. Syed Ali and J. Yogambigai, "Synchronization criterion of complex dynamical networks with both leakage delay and coupling delay on time scales," Neural Processing Letters, vol. 49, no. 2, pp. 453-466, 2019.

[41] Z. Huang, J. Cao, and Y. N. Raffoul, "Hilger-type impulsive differential inequality and its application to impulsive synchronization of delayed complex networks on time scales," Science China Information Sciences, vol. 61, no. 7, pp. 1-3, 2018.

[42] X. Lu, X. Zhang, and Q. Liu, "Finite-time synchronization of nonlinear complex dynamical networks on time scales via pinning impulsive control," Neurocomputing, vol. 275, pp. 2104$2110,2018$.

[43] Q. Xiao, F. L. Lewis, and Z. Zeng, "Event-based time-interval pinning control for complex networks on time scales and applications," IEEE Transactions on Industrial Electronics, vol. 65, no. 11, pp. 8797-8808, 2018.

[44] M. Bohner and A. Peterson, Dynamic Equations on Time Scales: An Introduction with Applications, Birkhäuser, Boston, 2001.

[45] M. Bohner and A. Peterson, Advances in Dynamic Equations on Time Scales, Birkhäuser, Boston, 2003.

[46] A. N. Langville and W. J. Stewart, "The Kronecker product and stochastic automata networks," Journal of Computational and Applied Mathematics, vol. 167, no. 2, pp. 429-447, 2004.

[47] Q. Cheng and J. Cao, "Global synchronization of complex networks with discrete time delays and stochastic disturbances," Neural Computing \& Applications, vol. 20, no. 8, pp. 1167-1179, 2011.

[48] H. Lütkepohl, Handbook of Matrices, Wiley, New York, NY, USA, 1996.

[49] L. O. Chua and D. N. Green, "A qualitative analysis of the behavior of dynamic nonlinear networks: stability of autonomous networks," IEEE Transactions on Circuits and Systems, vol. 23, no. 6, pp. 355-379, 1976. 